\title{
Profiles of anemia in adolescent students with sports club membership in an outpatient clinic setting
} \author{
Kitamura $^{7}$, Yoshinobu Takemoto ${ }^{2}$ \\ ${ }^{1}$ Department of Internal Medicine, University of Tokyo, Minato, Tokyo, Japan \\ 2 Department of Internal Medicine, Yoshinobu Clinic, Kagoshima, Kagoshima, Japan \\ 3 Department of Internal Medicine, Navitas Clinic Tachikawa, Tachikawa, Tokyo, Japan \\ 4 Department of Internal Medicine, Medical Governance Research Institute, Minato, Tokyo, Japan \\ 5 Department of Radiation Health Management, Fukushima Medical University, Fukushima, Fukushima, Japan \\ 6 Department of Internal Medicine, Navitas Clinic Kawasaki, Kawasaki, Kanagawa, Japan \\ 7 Division of Cellular Therapy, The Institute of Medical Science, The University of Tokyo, Minato, Tokyo, Japan \\ Corresponding Author: Kana Yamamoto \\ Email address: kanachan.y.0508@gmail.com
}

Kana Yamamoto ${ }^{\text {Corresp., } 1,2}{ }^{2}$, Morihito Takita ${ }^{3}$, Masahiro Kami ${ }^{4}$, Masaharu Tsubokura ${ }^{5}$, Tetsuya Tanimoto ${ }^{6}$, Toshio

Background. Anemia is a common health issue among adolescents. Anemic conditions could affect physical performance; however, the actual profiles of anemia in adolescent students in sports clubs have not been well documented. Methods. We conducted a retrospective chart review of individuals aged 13-22 years who belonged to sports clubs in schools and visited an outpatient clinic between August 1, 2016, and August 31, 2020. The medical and laboratory records, including serum levels of ferritin, folate, vitamin B12, and creatinine kinase at their first visit were assessed. Results. A total of 485 individuals (231 male [48\%] and 254 female [52\%] patients) were eligible for the study. The most common club activity was track and field ( $n=171$ [35\%]). The overall prevalence of the World Health Organization-defined anemia was 16.5\% (95\% confidence interval: 13.1-20.4; 9.0\% [5.4-13.8] and 23.1\% [17.8 - 29.2] in males and females, respectively) after excluding pretreated individuals. Hypoferritinemia and elevation of serum creatinine kinase levels were identified as independent contributors to anemia in both sexes (odds ratios: 13.2 [95\% confidence interval: 4.2-41.1], $p<0.001$ and 14.7 [95\% confidence interval: 1.8-118.4], $p$ $=0.012$, respectively for males; odds ratios: 6.6 [95\% confidence interval: $1.3-13.9$ ], $p$ $<0.001$ and 2.7 [95\% confidence interval: 1.4-5.5], $p=0.004$, respectively for females). Discussion. Anemia is prevalent in both male and female adolescent students in sports clubs. Iron deficiency and excessive training indicated by elevated creatinine kinase levels may contribute to the risk of anemia. Physicians should assess the amount of exercise, and not merely iron storage, in clinical practice. 


\section{Manuscript Title}

\section{Profiles of anemia in adolescent students with sports club}

\section{3 membership in an outpatient clinic setting}

\section{Authors}

6 Kana Yamamoto ${ }^{1,2}, \mathrm{MD}$, Morihito Takita $^{3}, \mathrm{MD}$, Masahiro Kami ${ }^{4}, \mathrm{MD}$, Masaharu Tsubokura ${ }^{5}$,

7 MD, Tetsuya Tanimoto ${ }^{6}, \mathrm{MD}$, Toshio Kitamura ${ }^{7}, \mathrm{MD}$, Yoshinobu Takemoto ${ }^{2}, \mathrm{MD}$ 8

\section{Affiliations}

$10{ }^{1}$ Department of Internal Medicine, University of Tokyo, Minato, Tokyo, Japan.

$11{ }^{2}$ Department of Internal Medicine, Yoshinobu Clinic, Kagoshima, Kagoshima, Japan.

$12{ }^{3}$ Department of Internal Medicine, Navitas Clinic Tachikawa, Tachikawa, Tokyo, Japan.

13 4 Department of Internal Medicine, Medical Governance Research Institute, Minato, Tokyo, 14 Japan.

15 5epartment of Radiation Health Management, Fukushima Medical University, Fukushima, 16 Fukushima, Japan.

$17{ }^{6}$ Department of Internal Medicine, Navitas Clinic Kawasaki, Kawasaki, Kanagawa, Japan.

$18{ }^{7}$ Division of Cellular Therapy, The Institute of Medical Science, The University of Tokyo,

19 Minato, Tokyo, Japan.

\section{Corresponding author}

22 Kana Yamamoto, MD.

23 Department of Internal Medicine, University of Tokyo, 4-6-1 Shirokanedai, Minato, Tokyo, 10824 0071, Japan.

\section{E-mail: kanachan.y.0508@gmail.com}




\section{Abstract}

28 Background. Anemia is a common health issue among adolescents. Anemic conditions could 29 affect physical performance; however, the actual profiles of anemia in adolescent students in 30 sports clubs have not been well documented.

32 Methods. We conducted a retrospective chart review of individuals aged 13-22 years who belonged to sports clubs in schools and visited an outpatient clinic between August 1, 2016, and August 31,2020. The medical and laboratory records, including serum levels of ferritin, folate, vitamin B12, and creatinine kinase at their first visit were assessed.

Results. A total of 485 individuals (231 male [48\%] and 254 female [52\%] patients) were eligible for the study. The most common club activity was track and field $(n=171[35 \%])$. The overall prevalence of the World Health Organization-defined anemia was 16.5\% (95\% confidence interval: $13.1-20.4 ; 9.0 \%$ [5.4-13.8] and 23.1\% [17.8-29.2] in males and females, respectively) after excluding pre-treated individuals. Hypoferritinemia and elevation of serum creatinine kinase levels were identified as independent contributors to anemia in both sexes 43 (odds ratios: 13.2 [95\% confidence interval: 4.2-41.1], $p<0.001$ and 14.7 [95\% confidence interval: $1.8-118.4$ ],$p=0.012$, respectively for males; odds ratios: 6.6 [95\% confidence interval: 1.3-13.9], $p<0.001$ and 2.7 [95\% confidence interval: 1.4-5.5], $p=0.004$, respectively for 46 females).

48 Discussion. Anemia is prevalent in both male and female adolescent students in sports clubs. Iron deficiency and excessive training indicated by elevated creatinine kinase levels may contribute to the risk of anemia. Physicians should assess the amount of exercise, and not merely 51 iron storage, in clinical practice. 


\section{Introduction}

54

55 Anemia is a medical condition commonly observed in clinical practice, in which the number of 56 red blood cells and, consequently, their oxygen-carrying capacity are insufficient to meet the physiological demands (WHO 2011). It causes weakness, fatigue, difficulty concentrating, poor school performance, and decreased work productivity due to non-specific symptoms ascribed to the diminished levels of oxygen delivered to the body tissues. Anemia can be classified into three categories: defects in marrow production, red cell immaturity, and decreased red cell survival.

The most common cause of anemia is iron deficiency (ID), which causes a reduction in red cell production in the marrow (Pasricha et al. 2021). The clinical features of ID anemia (IDA) vary with sex and age. In adolescents, anemia is more common in females than in males. A metaanalysis of national surveys estimated the prevalence of IDA in women of pre-school and reproductive ages as 9.6\% and 10.8\%, respectively (Petry et al. 2016). Diet and lifestyle effects, owing to the desire for thinness in young women, and menstrual bleeding have been identified as the causes of IDA (Fayet et al. 2014). In one study, , no significant sex difference was observed among older adults, and the prevalence of IDA was 12\% (Price et al. 2011). The main causes of IDA include gastric cancer, peptic ulcers, gastrectomy, and aspirin medication (Vetrano et al. 71 2020).

Anemia is a serious problem among athletes (McCormick et al. 2020). The prevalence rates of IDA in professional and elite athletes were reported to be $2 \%-29 \%$ and $3 \%-7 \%$ in women (Landahl et al. 2005; Ponorac et al. 2020) and men (Clement et al. 1987; Dubnov \& Constantini 2004), respectively. The primary mechanisms by which sports lead to ID are increased iron loss and blockage of iron absorption due to hepcidin bursts (Clenin et al. 2015). Anemic conditions may affect the physical performance, and ID in anemia limits the transport of oxygen. Notably, ID without anemia also influences the oxygenation capacity. Hence, the athletes should be given regular opportunities to undergo clinical laboratory assessments, correction of nutritional iron

81 intake, and nutritional fortification (Sacirovic et al. 2013). The International Olympic Committee

822009 Consensus Statement on periodic health evaluation of elite athletes recommends routine 83 screening for ID (Ljungqvist et al. 2009). 
84

85 ID occasionally causes problems other than anemia since iron is a major component of

86

87

88

89

90

91

92

myoglobin, which is a protein found in the muscles (Sim et al. 2019). Athletes could easily become iron deficient due to their high volume of muscle mass if they are not supplemented by adequate iron. Some studies have demonstrated the beneficial effects of iron administration for the treatment of chronic heart failure and ID, even in the absence of anemia (Anker et al. 2009; Okonko et al. 2008). The prevalence rates of ID were reported to be $49 \%-52 \%$ in female professional athletes (Nabhan et al. 2020; Ponorac et al. 2020), and 2.9\%-15\% in male college or elite athletes (Nabhan et al. 2020; Parks et al. 2017). Some researchers have attempted to provide iron supplements in athletes without anemia to improve their athletic performance. An Australian team reported that intravenous iron supplementation within 6 weeks of training improved the perceived fatigue and mood in distance runners without clinical ID (Woods et al. 2014).

In addition, the following mechanisms have been demonstrated to cause anemia in athletes: inadequate iron intake, intravascular hemolysis due to excessive exercise, gastrointestinal bleeding, exercise-induced acute phase response with production of inflammatory cytokines, dilution due to increased plasma volume, and insufficient intake of vitamins (Mougios 2007; Shaskey \& Green 2000; Williams 1989). These physiological responses have been suggested mainly in case reports or small retrospective studies, and the focus of current research was to verify their actual contribution to the development of anemia.

Most studies on anemia in athletes have focused on professional athletes or members of college athletic teams; however, little is known about the characteristics of anemia in young people who enjoy sports as part of school or community club activities. We have established an outpatient clinic specializing in anemia (Takemoto 2017; Takemoto 2019) to comprehensively assess the clinical laboratory data on anemia, including serum levels of haptoglobin, creatine kinase, folate, and vitamin $\mathrm{B} 12$ in addition to hemoglobin $(\mathrm{Hb})$ and ferritin as a fundamental assessment for the athletes. This study aimed to clarify the clinical characteristics of anemia in adolescent athletes. We retrospectively reviewed the medical and laboratory records of middle school-aged to college athletes between 13 and 22 years old.

Peer) reviewing PDF | (2021:10:66556:1:0:NEW 27 Jan 2022) 


\section{Materials and Methods}

\section{Study participants and design}

117 This study included patients between 13 and 22 years old who were affiliated with athletic clubs

118 in schools and visited our outpatient clinic that specializes in the treatment of patients with

119 anemia (Yoshinobu Clinic, Kagoshima, Japan) between August 1, 2016, and August 31, 2020.

120 The participants visited our clinic due to complaints of anemia symptoms or following the advice

121 from the trainers of their affiliated clubs. All the outpatients responded to the screening survey,

122 including questions on sports club activities, at the first visit, which facilitated the selection of

123 study participants.

124 The laboratory parameters measured using the blood samples of outpatients included the

125 complete blood cell count and serum levels of ferritin, vitamin B12, folate, creatine

126 phosphokinase, and haptoglobin. The serum zinc concentrations were measured after October

127 2019. Peripheral blood was collected and transported to a contract laboratory for measurement

128 (Kagoshima City Medical Association Laboratory Center, Kagoshima, Japan). The patients

129 answered the medical questionnaires containing questions related to their affiliated club,

130 frequency of practice, menstruation (for female patients), and iron supplementation.

131 We retrospectively collected and analyzed the clinical data after approval from the Institutional

132 Review Board (IRB) of the Medical Governance Research Institute (Tokyo, Japan) (approval

133 number: MG2020-08). The requirement for obtaining informed consent was waived, and the

134 consent was secured in an opt-out manner because of the retrospective and observational nature

135 of this study in accordance with the Ethical Guidelines for Medical and Health Research

136 Involving Human Subjects in Japan.

\section{Definitions}

139 In our study, anemia was defined as an $\mathrm{Hb}$ level of $\leq 12.0 \mathrm{~g} / \mathrm{dL}$ in both male and female patients 140 aged $\leq 14$ years, or lower than $13.0 \mathrm{~g} / \mathrm{dL}$ in men aged $>15$ years, in accordance with the criteria 141 of the World Health Organization (WHO) (WHO 2011). ID was defined as a serum ferritin level 142 of $\leq 30 \mu \mathrm{g} / \mathrm{L}$ (Camaschella 2015). The cutoff values of serum haptoglobin levels were 83, 66, 143 and $25 \mathrm{mg} / \mathrm{dL}$ for phenotypes 1-1, 2-1, and 2-2, respectively, in accordance with the reference 144 range of the testing laboratory. The cutoff values for serum levels of creatinine kinase, vitamin $145 \mathrm{~B} 12$, and folate were $147 \mathrm{IU} / \mathrm{L}, 350 \mathrm{pg} / \mathrm{mL}$, and $4 \mathrm{ng} / \mathrm{mL}$, respectively. The mean corpuscular 
146 volume $(\mathrm{MCV})$ and mean corpuscular hemoglobin $(\mathrm{MCH})$ were calculated based on the

147 complete blood cell count (Reddy \& Morlote 2021).

148

\section{Statistical analysis}

150 The participants' characteristics were summarized using descriptive statistics. The two-sided

$15195 \%$ confidence interval (CI) of a proportion was determined using the Clopper-Pearson

152 method. Two-group comparisons were performed using the Mann-Whitney U test for continuous

153 variables and Fisher's exact test for categorical variables. Multivariate logistic regression models

154 were employed to identify the patient characteristics associated with the risk of anemia.

155 Multivariate analysis was performed using a stepwise backward selection with the Wald method

156 for significant variables in the univariate assessment $(p<0.05)$. Statistical significance was

157 considered when the two-sided $p$ value was less than 0.05 . All statistical analyses were

158 performed using SPSS version 27 (IBM, Armonk, NY, USA).

159

160

Results

161 Patient characteristics

162 The patient characteristics ( $n=485$ in total, 231 male [48\%] and 254 female [52\%] patients) are

163 shown in Table 1. The median age was 15 years ([range,13-22] years). Nine females (4\%) had

164 not yet experienced their first menstruation, and 72 (28\%) females reported irregular

165 menstruation in the self-reported screening survey. The most common club membership was

166 Track and Field/Athletics $(n=171,35 \%)$, in which 101 (55\%) practiced middle- or long-distance

167 running. A total of 55 patients (11\% of the total cohort) took either iron medication prescribed by

168 physicians from other clinics or commercially available iron supplements (Figure 1). We

169 excluded them from further analysis and focused on non-treated individuals.

170

171 Non-treated cohort

172 Anemia, as defined by the WHO criteria, was observed in 71 patients (16.5\% [95\% CI: 13.1-

$17320.4])$, consisting of 18 males (9.0\% [95\% CI: 5.4-13.8]) and 53 females (23.1\% [95\% CI: 17.8-

174 29.2]), in the non-treated cohort at initial examination $(n=430)$ (Table 2$)$. No significant

175 differences were observed in the patient characteristics between the anemic and non-anemic

176 groups in both males and females. The anemia group, however, showed significantly higher

Peer] reviewing PDF | (2021:10:66556:1:0:NEW 27 Jan 2022) 
177 proportions of $\mathrm{MCV}(80 \mathrm{fL}), \mathrm{MCH}(27 \mathrm{pg})$, and hypoferritinemia than the non-anemic group in

178 both sex groups $(p=0.001,<0.001$, and $<0.001$ for males, and $<0.001,<0.001$, and $<0.001$

179 for females, respectively). Elevated serum creatinine kinase levels were more frequently

180 observed in the anemic group than in the non-anemic groups in both men and women $(p=0.002$

181 and 0.003 , respectively). Anemic females exhibited a significantly higher prevalence of folate

182 deficiency than non-anemic females $(p=0.006)$. No significant differences were observed in the

183 prevalence of zinc deficiency between the anemic and non-anemic groups in either sex.

184

185 Iron deficiency was identified in 55 patients (77.5\% in anemic patients) (13 male [72.2\%] and 42

186 female [79.2\%] patients). Then, the laboratory data of anemic patients with and without ID were

187 compared (Supplemental Table 1). Significantly lower Hb, MCV, and MCH were found in the

188 IDA group than in the non-IDA group in both sexes $(p=0.007,0.03$, and 0.003 for males, and

$1890.001,0.003$, and 0.001 for females, respectively). A significantly higher prevalence of vitamin

190 B12 deficiency was observed in males with IDA than in males without IDA (69\% versus $0 \%, p=$

191 0.029). The Hb levels in anemic patients without ID in both sexes were close to the lower limits

192 of the reference range.

193

194 Predictors for anemic status

195 Results of the univariate logistic regression analysis of the factors associated with the

196 development of anemia in the male cohort revealed that belonging to the Track and

197 Field/Athletics club, hypoferritinemia, and elevated creatinine kinase were significant predictors

198 ( $p=0.036, p<0.001$, and $p=0.020$, respectively) (Supplemental Table 2). Results of the

199 multivariate analysis conducted in males revealed hypoferritinemia (odds ratio: 13.2 [95\% CI:

$2004.2-41.1]$ for adjusted odds ratio, $p<0.001$ ) and elevation of creatinine kinase level (odds ratio:

20114.7 [95\% CI: 1.8-118.4], $p=0.012$ ) as independent predictors (Table 3). In females,

202 hypoferritinemia and elevated creatinine kinase levels were also identified as independent

203 predictors after conducting a multivariate regression analysis followed by a univariate

204 assessment (6.6 [1.3-13.9], $p<0.001$, and 2.7 [1.4-5.5], $p=0.004$, respectively).

205

206 Pre-treated patients with iron supplementation

207 A total of 55 participants, consisting of 30 males and 25 females, were treated with iron 
208 supplements, either using the prescribed medication from other outpatient clinics or over-the-

209 counter (OTC) products before their initial presentation at our clinic. Twelve patients (six males

210 and six females) exhibited symptoms of WHO-defined anemia despite receiving iron

211 supplementation (Table 4). IDA was observed in seven of these patients (three males and four

212 females). Eight patients (five males and three females) were administered with prescribed iron

213 medication, while five presented with vitamin B12 deficiency.

214

\section{Discussion}

216 This study provides valuable information to physicians treating adolescent athletes since a set of

217 comprehensive laboratory examinations including vitamin B12, Zn, haptoglobin, folate, and

218 creatine kinase tests in addition to $\mathrm{Hb}$ and ferritin tests were performed as routine examinations.

219 Notably, anemia was identified in $9 \%$ and $23 \%$ of males and females in our cohort, respectively.

220 The prevalence of anemia in this study was higher than that in a previous study, in which $3.5 \%$

221 of boys and $8.1 \%$ of girls aged 14 to 17 years belonging in sports clubs were diagnosed with

222 anemia (Toivo et al. 2020). Females are more susceptible to anemia than males due to menstrual

223 blood loss. A few available studies have evaluated the prevalence of anemia in male athletes of

224 middle and high school age. Anemia is an important issue in adolescent athletes, regardless of

225 sex.

226

227 ID is the most common cause of anemia in both males and females. In fact, $72 \%$ and $79 \%$ of

228 anemic males and females, respectively, had IDA in this study. Insufficient iron intake, dietary

229 restriction, menstruation, bleeding from the gastrointestinal tract, and iron loss through sweating

230 are known causes of IDA in men and women, especially committed athletes (McClung 2012).

231 However, iron intake and iron loss through sweating or gastrointestinal bleeding were not

232 evaluated in this study, which will be the focus of a future study to identify the determinants of

233 IDA in adolescent athletes. Anemic participants without ID presented mild anemia close to the

234 lower limit of our reference range: $\mathrm{Hb}$ levels of $12.8 \mathrm{~g} / \mathrm{dL}$ (range: 11.7-12.9) and $11.8 \mathrm{~g} / \mathrm{dL}$

235 (11.0-11.9) in males and females, respectively. This finding suggests that the clinical

236 significance of anemia without ID may be minor; however, a hematopoietic disorder, such as

237 aplastic anemia, should be ruled out.

238 
239 Overtraining may be a risk factor for anemia in adolescent athletes. Approximately $40 \%$ of the

240 participants presented low haptoglobin levels regardless of anemia and sex classification.

241 Declining haptoglobin levels, in general, suggest the presence of chronic hemolysis, although

242 serum free $\mathrm{Hb}$ levels as direct evidence of hemolysis were not assessed. In the anemia group,

$24394 \%$ and $49 \%$ of males and females, respectively showed elevated creatine kinase levels, while

$24486 \%$ and $89 \%$ of males and females, respectively practiced in the club for more than 5 days per

245 week. The cause of hemolysis is exercise-induced intravascular hemolysis, which is commonly

246 observed in long-distance runners (Lippi \& Sanchis-Gomar 2019). Excessive exercise is

247 associated with bleeding in the digestive tract. The most common club membership in our cohort

248 was Track \& Field/Athletics, accounting for 35\%. Among the Track \& Field/Athletics club

249 members, 59\% specialized in middle- or long-distance running. These results support the

250 association between chronic hemolysis and excessive training although the intensity and volume

251 of their training were lack to analyze in this study. Instructors of club sports should pay attention

252 to the amount of practice to prevent overtraining, which may increase the risk of injury and

253 anemia in young athletes.

254

255

Another possible contributor to the development of anemia in adolescent athletes is hepcidin

256

257

258

259

260

261

262

263 The sub-analysis of anemic patients showed the clinical importance of vitamin B12 metabolism 264

265

266

267

268

269

response, which regulates iron homeostasis (Camaschella 2015). Hepcidin stimulation decreases iron absorption in the intestine and promotes iron sequestration in tissues. An exercise-induced inflammatory response, which is indicated by elevated serum interleukin- 6 levels, was associated with hepcidin expression (Newlin et al. 2012). Thus, alterations in hepcidin anemic athletes could be observed if hepcidin levels were measured. Future studies will include the assessment of hepcidin levels. when physicians consider the treatment of IDA. Approximately half of the participants, ranging between 47\% and 56\%, showed vitamin B12 deficiency across sexes and anemia conditions. Furthermore, a significantly higher proportion of males with IDA developed vitamin B12 deficiency compared with those without ID $(69 \%$ versus $0 \%, p=0.03)$. Vitamin B12 is an essential nutrient for the formation and maturation of red blood cells (Oh \& Brown 2003). Hemopoiesis due to exercise increases the demand for vitamin B12 (Koury \& Ponka 2004).

Peer) reviewing PDF | (2021:10:66556:1:0:NEW 27 Jan 2022) 
270 Insufficient intake and dietary restrictions in athletes who are trying to control their weight may

271 also cause vitamin B12 deficiency (Economos et al. 1993). Young athletes are at high risk of

272 developing anemia due to vitamin B12 deficiency, in addition to ID.

273

274 Iron supplementation alone may not be sufficient for treating anemia in adolescent athletes.

275 Interestingly, we identified vitamin B12 deficiency in five out of eight study participants who

276 were already taking prescribed iron medications. Most OTC conventional iron preparations

277 contain vitamin B12 and folic acid. Physicians commonly prescribe iron agents alone for the

278 treatment of IDA. This study highlights the potential beneficial impact of vitamin B12

279 supplementation in addition to iron intake.

280

281 Our study can be considered significant as it reports the prevalence of anemia in young athletes

282 by providing comprehensive clinical laboratory data; however, the study has some limitations.

283 First, it was a retrospective analysis of the data on individuals who were considered or suspected

284 of having anemia. Therefore, the results of this study cannot be generalized because they could

285 overestimate the prevalence of anemia. Second, there may be unrecognized bias due to the small

286 sample size. For example, many participants were affiliated with the Track \& Field/Athletics

287 club, soccer/football club, and basketball club. These athletes and their coaches may be paying

288 more attention to anemia in light of recent news reports on the overtreatment of anemia with iron

289 injection (Kobayashi et al. 2019).

290

291 Conclusion

292 Anemia is common in both male and female young athletes. ID and the negative effects of

293 intensive or excessive training, as indicated by elevated levels of serum creatinine kinase, could

294 contribute to the development of anemia. Vitamin B12 deficiency may be a clinical pitfall for

295 treatment. Physicians treating adolescent athletes who present with anemia should pay attention

296 to the amount and type of training and vitamin B12 metabolism, in addition to iron

297 supplementation.

298

299 Acknowledgments

300 We express our gratitude to medical and administrative staff at the Yoshinobu Clinic 
301 (Kagoshima, Japan) and Ms. Erika Yamashita (Medical Governance Research Institute, Tokyo, 302 Japan) for their assistance of data collection and Editage service for the proofreading. 303 
304

305

306

307

308

309

310

311

312

313

314

315

316

317

318

319

320

321

322

323

324

325

326

327

328

329

330

331

332

333

334

335

336

337

338

339

340

341

342

343

344

345

346

347

348

\section{References}

Anker SD, Comin Colet J, Filippatos G, Willenheimer R, Dickstein K, Drexler H, Luscher TF, Bart B, Banasiak W, Niegowska J, Kirwan BA, Mori C, von Eisenhart Rothe B, Pocock SJ, Poole-Wilson PA, Ponikowski P, and Investigators F-HT. 2009. Ferric carboxymaltose in patients with heart failure and iron deficiency. $N$ Engl J Med 361:2436-2448. 10.1056/NEJMoa0908355

Camaschella C. 2015. Iron-deficiency anemia. N Engl J Med 372:1832-1843. 10.1056/NEJMra1401038

Clement DB, Lloyd-Smith DR, Macintyre JG, Matheson GO, Brock R, and Dupont M. 1987. Iron status in Winter Olympic sports. J Sports Sci 5:261-271. 10.1080/02640418708729781

Clenin G, Cordes M, Huber A, Schumacher YO, Noack P, Scales J, and Kriemler S. 2015. Iron deficiency in sports - definition, influence on performance and therapy. Swiss Med Wkly 145:w14196. 10.4414/smw.2015.14196

Dubnov G, and Constantini NW. 2004. Prevalence of iron depletion and anemia in top-level basketball players. Int J Sport Nutr Exerc Metab 14:30-37. 10.1123/ijsnem.14.1.30

Economos CD, Bortz SS, and Nelson ME. 1993. Nutritional practices of elite athletes. Practical recommendations. Sports Med 16:381-399. 10.2165/00007256-199316060-00004

Fayet F, Flood V, Petocz P, and Samman S. 2014. Avoidance of meat and poultry decreases intakes of omega-3 fatty acids, vitamin B12, selenium and zinc in young women. $J$ Hum Nutr Diet 27 Suppl 2:135-142. 10.1111/jhn.12092

Kobayashi Y, Tahara K, Ishii A, and Arai R. 2019. Athletics federation to ban iron shots by long-distance runners after risks become clear. Available at https://mainichi.jp/english/articles/20190102/p2a/00m/0sp/006000c (accessed 10/1/2021 2021).

Koury MJ, and Ponka P. 2004. New insights into erythropoiesis: the roles of folate, vitamin B12, and iron. Annu Rev Nutr 24:105-131. 10.1146/annurev.nutr.24.012003.132306

Landahl G, Adolfsson P, Börjesson M, Mannheimer C, and Rödjer S. 2005. Iron deficiency and anemia: a common problem in female elite soccer players. Int J Sport Nutr Exerc Metab 15:689-694. 10.1123/ijsnem.15.6.689

Lippi G, and Sanchis-Gomar F. 2019. Epidemiological, biological and clinical update on exercise-induced hemolysis. Ann Transl Med 7:270. 10.21037/atm.2019.05.41

Ljungqvist A, Jenoure P, Engebretsen L, Alonso JM, Bahr R, Clough A, De Bondt G, Dvorak J, Maloley R, Matheson G, Meeuwisse W, Meijboom E, Mountjoy M, Pelliccia A, Schwellnus M, Sprumont D, Schamasch P, Gauthier JB, Dubi C, Stupp H, and Thill C. 2009. The International Olympic Committee (IOC) Consensus Statement on periodic health evaluation of elite athletes March 2009. Br J Sports Med 43:631-643. 10.1136/bjsm.2009.064394

McClung JP. 2012. Iron status and the female athlete. J Trace Elem Med Biol 26:124-126. 10.1016/j.jtemb.2012.03.006

McCormick R, Sim M, Dawson B, and Peeling P. 2020. Refining Treatment Strategies for Iron Deficient Athletes. Sports Med 50:2111-2123. 10.1007/s40279-020-01360-2

Mougios V. 2007. Reference intervals for serum creatine kinase in athletes. Br J Sports Med 41:674-678. 10.1136/bjsm.2006.034041 
349

350

351

352

353

354

355

356

357

358

359

360

361

362

363

364

365

366

367

368

369

370

371

372

373

374

375

376

377

378

379

380

381

382

383

384

385

386

387

388

389

390

391

392

393

394

Nabhan D, Bielko S, Sinex JA, Surhoff K, Moreau WJ, Schumacher YO, Bahr R, and Chapman RF. 2020. Serum ferritin distribution in elite athletes. J Sci Med Sport 23:554-558. 10.1016/j.jsams.2019.12.027

Newlin MK, Williams S, McNamara T, Tjalsma H, Swinkels DW, and Haymes EM. 2012. The effects of acute exercise bouts on hepcidin in women. Int J Sport Nutr Exerc Metab 22:79-88. 10.1123/ijsnem.22.2.79

Oh R, and Brown DL. 2003. Vitamin B12 deficiency. Am Fam Physician 67:979-986.

Okonko DO, Grzeslo A, Witkowski T, Mandal AK, Slater RM, Roughton M, Foldes G, Thum T, Majda J, Banasiak W, Missouris CG, Poole-Wilson PA, Anker SD, and Ponikowski P. 2008. Effect of intravenous iron sucrose on exercise tolerance in anemic and nonanemic patients with symptomatic chronic heart failure and iron deficiency FERRIC-HF: a randomized, controlled, observer-blinded trial. J Am Coll Cardiol 51:103-112. 10.1016/j.jacc.2007.09.036

Parks RB, Hetzel SJ, and Brooks MA. 2017. Iron Deficiency and Anemia among Collegiate Athletes: A Retrospective Chart Review. Med Sci Sports Exerc 49:1711-1715. 10.1249/MSS.0000000000001259

Pasricha SR, Tye-Din J, Muckenthaler MU, and Swinkels DW. 2021. Iron deficiency. Lancet 397:233-248. 10.1016/S0140-6736(20)32594-0

Petry N, Olofin I, Hurrell RF, Boy E, Wirth JP, Moursi M, Donahue Angel M, and Rohner F. 2016. The Proportion of Anemia Associated with Iron Deficiency in Low, Medium, and High Human Development Index Countries: A Systematic Analysis of National Surveys. Nutrients 8. 10.3390/nu8110693

Ponorac N, Popovic M, Karaba-Jakovljevic D, Bajic Z, Scanlan A, Stojanovic E, and Radovanovic D. 2020. Professional Female Athletes Are at a Heightened Risk of IronDeficient Erythropoiesis Compared With Nonathletes. Int J Sport Nutr Exerc Metab 30:48-53. 10.1123/ijsnem.2019-0193

Price EA, Mehra R, Holmes TH, and Schrier SL. 2011. Anemia in older persons: etiology and evaluation. Blood Cells Mol Dis 46:159-165. 10.1016/j.bcmd.2010.11.004

Sacirovic S, Asotic J, Maksimovic R, Radevic B, Muric B, Mekic H, and Biocanin R. 2013. Monitoring and prevention of anemia relying on nutrition and environmental conditions in sports. Mater Sociomed 25:136-139. 10.5455/msm.2013.25.136-139

Shaskey DJ, and Green GA. 2000. Sports haematology. Sports Med 29:27-38. 10.2165/00007256-200029010-00003

Sim M, Garvican-Lewis LA, Cox GR, Govus A, McKay AKA, Stellingwerff T, and Peeling P. 2019. Iron considerations for the athlete: a narrative review. Eur J Appl Physiol 119:1463-1478. 10.1007/s00421-019-04157-y

Takemoto Y. 2017. The mystery of mean corpuscular volume. Journal of The Kagoshima City Medical Association 56:66-67.

Takemoto Y. 2019. The lower level of serum vitamin B12 without increase in the mean corpuscular volume in summer. Journal of The Kagoshima City Medical Association 58:22-25.

Toivo K, Kannus P, Kokko S, Alanko L, Heinonen OJ, Korpelainen R, Savonen K, Selanne H, Vasankari T, Kannas L, Kujala UM, Villberg J, Niemela O, and Parkkari J. 2020. Haemoglobin, iron status and lung function of adolescents participating in organised sports in the Finnish Health Promoting Sports Club Study. BMJ Open Sport Exerc Med 6:e000804. 10.1136/bmjsem-2020-000804 
395 V.B. Reddy V, and Morlote D. 2021. Examination of Blood and Marrow Cells. In: Kaushansky K, Prchal JT, Burns LJ, Lichtman MA, Levi M, and Linch DC, eds. Williams Hematology, 10e. New York, NY: McGraw-Hill Education.

Vetrano DL, Zucchelli A, Marconi E, Levi M, Pegoraro V, Cataldo N, Heiman F, Cricelli C, and Lapi F. 2020. Predictors of iron-deficiency anemia in primary care older adults: a realworld European multi-country longitudinal study. Aging Clin Exp Res 32:2211-2216. 10.1007/s40520-019-01454-6 severity. World Health Organization.

Williams MH. 1989. Vitamin supplementation and athletic performance. Int J Vitam Nutr Res Suppl 30:163-191.

Woods A, Garvican-Lewis LA, Saunders PU, Lovell G, Hughes D, Fazakerley R, Anderson B, Gore CJ, and Thompson KG. 2014. Four weeks of IV iron supplementation reduces perceived fatigue and mood disturbance in distance runners. PLoS One 9:e108042.

410 10.1371/journal.pone.0108042 
Figure 1

Study cohort profile.

The procedures on inclusion of this study cohort is shown.

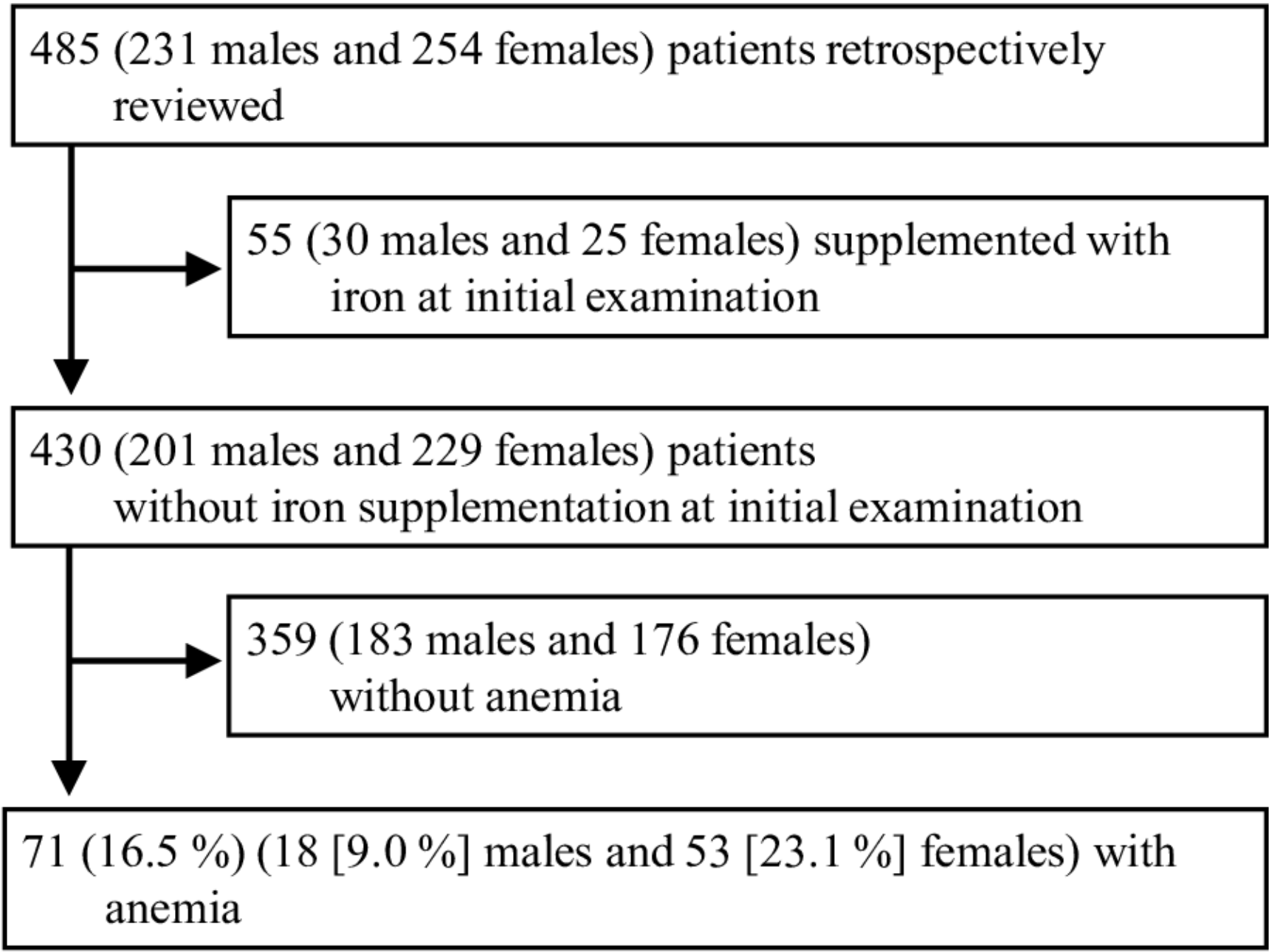


Table $\mathbf{1}$ (on next page)

Participant Characteristics.

Data were missing in $19 *$ and $231 \dagger$ cases, respectively. 


\begin{tabular}{ll}
\hline Variables & $\begin{array}{l}\text { Median [range] or } \\
\text { Number (percentage) }\end{array}$ \\
\hline Age (years) & $15[13-22]$ \\
Sex-female & $254(52)$ \\
Height (cm)* & $163(139-186)$ \\
Body weight (kg)* & $51(34-92)$ \\
Body mass index (kg/m²)* & $19.2(13.6-31.1)$ \\
Affiliated club & $171(35)$ \\
Track \& Field/Athletics & $105(22)$ \\
Basketball & $42(9)$ \\
Soccer/football & $27(6)$ \\
Volleyball & $26(5)$ \\
Tennis & $114(24)$ \\
Others & \\
Frequency of practice ${ }^{\dagger}$ &
\end{tabular}

1 


\section{Table 2 (on next page)}

Characteristics and laboratory data classified by sex and anemic status in non-treated participant group.

Median [range] or number (percentage) are shown. No missing data were presented except for menstruation (201 females included), frequency of practice (104 males and 113 females) and Zinc deficiency (68 males and 73 females). Abbreviation; BMI: body mass index, MCV: mean corpuscular volume, $\mathrm{MCH}$ : mean corpuscular hemoglobin. The Mann-Whitney U tests and Fisher's exact tests were performed for continuous and for categorical variables, respectively. 


\begin{tabular}{|c|c|c|c|c|c|c|}
\hline Variables & $\begin{array}{l}\text { Anemia } \\
(n=18)\end{array}$ & $\begin{array}{l}\text { Non-anemia } \\
(n=183)\end{array}$ & $\begin{array}{l}p \\
\text { value }\end{array}$ & $\begin{array}{l}\text { Anemia } \\
(n=53)\end{array}$ & $\begin{array}{l}\text { Non-anemia } \\
(n=176)\end{array}$ & $\begin{array}{l}p \\
\text { value }\end{array}$ \\
\hline \multicolumn{7}{|l|}{ Patient Characteristics } \\
\hline Age (years) & $16[13-17]$ & $15[13-22]$ & 0.300 & $15[13-22]$ & $15[13-22]$ & 0.414 \\
\hline Menstruation* & & & & & & 0.723 \\
\hline No menstruation & & & & $1(2)$ & $7(5)$ & \\
\hline Regular menstruation & & & & $31(65)$ & $99(65)$ & \\
\hline Track \& Field/Athletics & $11(61)$ & $75(41)$ & & $11(21)$ & $36(21)$ & \\
\hline Basketball & $2(11)$ & $17(9)$ & & $21(40)$ & $57(32)$ & \\
\hline Soccer/football & $4(22)$ & $28(15)$ & & $1(2)$ & $3(2)$ & \\
\hline Others & $1(6)$ & $63(34)$ & & $20(38)$ & $80(46)$ & \\
\hline Frequency of practice ${ }^{\dagger}$ & & & 0.213 & & & 0.344 \\
\hline Less than 5 times a week & $1(14)$ & $20(21)$ & & $3(11)$ & $14(17)$ & \\
\hline 5 times a week or more & $6(86)$ & $77(79)$ & & $25(89)$ & $71(84)$ & \\
\hline
\end{tabular}


Laboratory Data

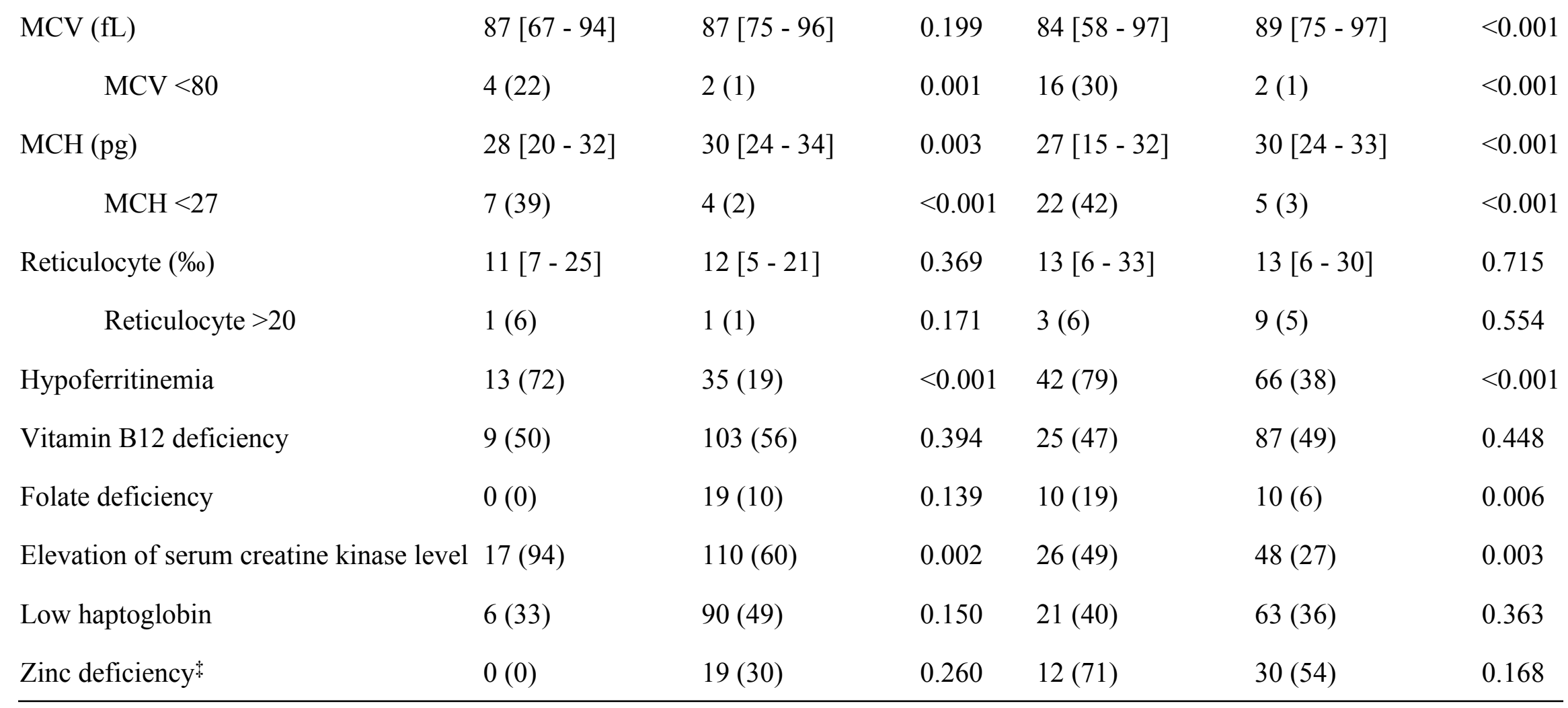




\section{Table 3(on next page)}

Multivariate analysis to predict anemia.

he adjusted odds ratios [95\% confidence interval] are shown. The Nagelkerke R-squares of multivariate logistic models were $33.7 \%$ and $23.2 \%$ for male and female, respectively. The $p$ values for the multivariate models were $<0.001$ for both sexes. 


\begin{tabular}{llllll}
\hline \multirow{2}{*}{ Variables } & \multicolumn{1}{l}{ Male } & & \multicolumn{2}{l}{ Female } \\
\cline { 2 - 3 } \cline { 5 - 6 } & Odds & $p$ value & Odds & $p$ value \\
\hline Hypoferritinemia & $13.2[4.2-41.1]$ & $<0.001$ & & $6.6[1.3-13.9]$ & $<0.001$ \\
Elevation of serum creatine kinase level & $14.7[1.8-118.4]$ & 0.012 & & $2.7[1.4-5.5]$ & 0.004 \\
\hline
\end{tabular}




\section{Table 4 (on next page)}

Case presentation of individuals who were already taking prescribed iron medications or self-treating iron supplementation.

Abbreviations: male; $M$, female; $F$, prescribed iron medication; $P$, self-treating over-thecounter iron supplementation; S, mean corpuscular volume; MCV, not identified for haptoglobin type; N, Mixed Martial Arts; MMA, Track \& Field/Athletcs; TFA, soccer/football; SF, basketball; B, volleyball; V. $\downarrow$ lower than reference ranges for levels of serum ferritin, vitamin B12, folate and haptoglobin. $\uparrow$ higher than reference range for creatinine kinase. 


\begin{tabular}{|c|c|c|c|c|c|c|c|c|c|c|c|c|}
\hline Case & 1 & 2 & 3 & 4 & 5 & 6 & 7 & 8 & 9 & 10 & 11 & 12 \\
\hline Sex & M & M & M & M & M & M & $\mathrm{F}$ & $\mathrm{F}$ & $\mathrm{F}$ & $\mathrm{F}$ & $\mathrm{F}$ & $\mathrm{F}$ \\
\hline $\begin{array}{l}\text { Type of pre- } \\
\text { existing iron } \\
\text { treatment }\end{array}$ & $\mathrm{P}$ & $\mathrm{P}$ & $\mathrm{P}$ & $\mathrm{P}$ & $\mathrm{P}$ & $\mathrm{S}$ & $\mathrm{P}$ & $\mathrm{P}$ & $\mathrm{P}$ & $\mathrm{S}$ & $\mathrm{S}$ & $\mathrm{S}$ \\
\hline Age (years) & 14 & 15 & 16 & 16 & 16 & 15 & 14 & 14 & 16 & 13 & 14 & 17 \\
\hline $\begin{array}{l}\text { Hemoglobin } \\
(\mathrm{g} / \mathrm{dL})\end{array}$ & 11.5 & 12.2 & 12.8 & 12.8 & 12.5 & 12.8 & 11.6 & 11.5 & 9.8 & 8.4 & 9.8 & 10 \\
\hline $\begin{array}{l}\text { Ferritin } \\
(\mu \mathrm{g} / \mathrm{L})\end{array}$ & $7.7 \downarrow$ & 68 & 50.1 & $22.3 \downarrow$ & $22.4 \downarrow$ & 83.1 & 110.1 & $9.9 \downarrow$ & 67 & $3.2 \downarrow$ & $6.7 \downarrow$ & $7.2 \downarrow$ \\
\hline MCV (fL) & 80 & 91 & 91 & 89 & 85 & 94 & 92 & 87 & 86 & 73 & 81 & 90 \\
\hline $\begin{array}{l}\text { Vitamin B12 } \\
(\mathrm{pg} / \mathrm{mL})\end{array}$ & $230 \downarrow$ & 458 & $291 \downarrow$ & $229 \downarrow$ & $215 \downarrow$ & $254 \downarrow$ & $225 \downarrow$ & 519 & 336 & 840 & 395 & 695 \\
\hline $\begin{array}{l}\text { Folate } \\
(\mathrm{ng} / \mathrm{mL})\end{array}$ & $3.8 \downarrow$ & 8.5 & 21.1 & 9.2 & 16.1 & 4.1 & 6.8 & 6.8 & 13 & 19 & 8.3 & 16.4 \\
\hline Creatine & & & & & & & & & & & & \\
\hline $\begin{array}{l}\text { Kinase } \\
(\mathrm{IU} / \mathrm{L})\end{array}$ & $187 \uparrow$ & $297 \uparrow$ & $450 \uparrow$ & $222 \uparrow$ & $157 \uparrow$ & $2622 \uparrow$ & 143 & $197 \uparrow$ & $235 \uparrow$ & 100 & $365 \uparrow$ & $467 \uparrow$ \\
\hline $\begin{array}{l}\text { Haptoglobin } \\
(\mathrm{mg} / \mathrm{dL})\end{array}$ & $\leq 10 \downarrow$ & $\leq 10 \downarrow$ & $42 \downarrow$ & 68 & $15 \downarrow$ & $\leq 10 \downarrow$ & 100 & $26 \downarrow$ & 30 & 29 & $15 \downarrow$ & $21 \downarrow$ \\
\hline
\end{tabular}




$\begin{aligned} & \begin{array}{l}\text { Haptoglobin- } \\ \text { Type }\end{array} \\ & \text { Club }\end{aligned}$
$\begin{aligned} & \text { MMA } \\ & \text { Membership }\end{aligned}$

